ANNALES

POLONICI MATHEMATICI

$92.3(2007)$

\title{
Some uniqueness results on meromorphic functions sharing three sets
}

by AbhiJit BAnerJee (Kalyani)

\begin{abstract}
We discuss the uniqueness of meromorphic functions when they share three sets with the notion of weighted sharing and improve two results of Lahiri-Banerjee and $\mathrm{Yi}-\mathrm{Lin}$. We also improve a recent result of the present author and thus provide an answer to a question of Gross, in a new direction.
\end{abstract}

1. Introduction, definitions and results. Let $f$ and $g$ be two nonconstant meromorphic functions defined in the open complex plane $\mathbb{C}$. We denote by $T(r)$ the maximum of $T(r, f)$ and $T(r, g)$. The notation $S(r)$ designates any quantity satisfying $S(r)=o(T(r))$ as $r \rightarrow \infty$, outside a possible exceptional set of finite linear measure.

If for some $a \in \mathbb{C} \cup\{\infty\}, f$ and $g$ have the same set of $a$-points with the same multiplicities then we say that $f$ and $g$ share the value a $C M$ (counting multiplicities). If we do not take the multiplicities into account, $f$ and $g$ are said to share the value a IM (ignoring multiplicities).

Let $S$ be a set of distinct elements of $\mathbb{C} \cup\{\infty\}$ and $E_{f}(S)=\bigcup_{a \in S}\{z$ : $f(z)-a=0\}$, where each zero is counted according to its multiplicity. If we do not count multiplicities the set $E_{f}(S)$ is denoted by $\bar{E}_{f}(S)$.

If $E_{f}(S)=E_{g}(S)$ we say that $f$ and $g$ share the set $S C M$. On the other hand if $\bar{E}_{f}(S)=\bar{E}_{g}(S)$, we say that $f$ and $g$ share the set $S I M$.

In [3] Gross posed the following question:

Can one find two finite sets $S_{j}(j=1,2)$ such that any two nonconstant entire functions $f$ and $g$ satisfying $E_{f}\left(S_{j}\right)=E_{g}\left(S_{j}\right)$ for $j=1,2$ must be identical?

Fang and $\mathrm{Xu}[2]$ considered the case of meromorphic functions and proved the following result.

2000 Mathematics Subject Classification: Primary 30D35.

Key words and phrases: meromorphic functions, uniqueness, weighted sharing, shared set. 
Theorem A ([2]). Let $S_{1}=\left\{z: z^{3}-z^{2}-1=0\right\}, S_{2}=\{0\}$ and $S_{3}=$ $\{\infty\}$. Suppose that $f$ and $g$ are two nonconstant meromorphic functions satisfying $\Theta(\infty ; f)>1 / 2$ and $\Theta(\infty ; g)>1 / 2$. If $E_{f}\left(S_{j}\right)=E_{g}\left(S_{j}\right)$ for $j=$ $1,2,3$ then $f \equiv g$.

Dealing with the question of Gross, Qiu and Fang [12] proved the following theorem.

Theorem B ([12]). Let $n \geq 3$ be a positive integer, $S_{1}=\left\{z: z^{n}-\right.$ $\left.z^{n-1}-1=0\right\}, S_{2}=\{0\}$, and let $f$ and $g$ be two nonconstant meromorphic functions whose poles are of multiplicities at least 2 . If $E_{f}(\{\infty\})=E_{g}(\{\infty\})$ and $E_{f}\left(S_{i}\right)=E_{g}\left(S_{i}\right)$ for $i=1,2$ then $f \equiv g$.

They also gave an example to show that the condition that the poles of $f$ and $g$ are of multiplicities at least 2 cannot be removed in Theorem B.

It should be noted that if two meromorphic functions $f$ and $g$ have no simple pole then clearly $\Theta(\infty ; f) \geq 1 / 2$ and $\Theta(\infty ; g) \geq 1 / 2$.

Lahiri and Banerjee [9] investigated the situation for $\Theta(\infty ; f) \leq 1 / 2$ and $\Theta(\infty, g) \leq 1 / 2$ in Theorem A and proved the following result.

Theorem C ([9]). Let $S_{1}=\left\{z: z^{n}+a z^{n-1}+b=0\right\}, S_{2}=\{0\}$ and $S_{3}=$ $\{\infty\}$, where $a, b$ are nonzero constants such that $z^{n}+a z^{n-1}+b=0$ has no multiple root and $n(\geq 4)$ is an integer. If for two nonconstant meromorphic functions $f$ and $g, E_{f}\left(S_{i}\right)=E_{g}\left(S_{i}\right)$ for $i=1,2,3$ and $\Theta(\infty ; f)+\Theta(\infty ; g)$ $>0$, then $f \equiv g$.

In $2004 \mathrm{Yi}$ and Lin [17] independently proved Theorem $\mathrm{C}$ assuming $\Theta(\infty ; f)>0$ instead of $\Theta(\infty ; f)+\Theta(\infty ; g)>0$. They remarked that the assumption $E_{f}\left(S_{2}\right)=E_{g}\left(S_{2}\right)$ in the above result can be relaxed to $\bar{E}_{f}\left(S_{2}\right)=\bar{E}_{g}\left(S_{2}\right)$.

Recently the present author [1] has investigated the relaxation of the nature of sharing the set $S_{1}$ in Theorem $\mathrm{C}$ using the idea of gradation of sharing of values and sets, known as weighted sharing, introduced in [6, 7]; it consists in measuring how close a shared value is to being shared IM or to being shared CM. We now give the definition.

DeFinition $1.1([6,7])$. Let $k$ be a nonnegative integer or infinity. For $a \in \mathbb{C} \cup\{\infty\}$ we denote by $E_{k}(a ; f)$ the set of all $a$-points of $f$, where an $a$-point of multiplicity $m$ is counted $m$ times if $m \leq k$ and $k+1$ times if $m>k$. If $E_{k}(a ; f)=E_{k}(a ; g)$, we say that $f, g$ share the value a with weight $k$.

We write $f, g$ share $(a, k)$ to mean that $f, g$ share the value $a$ with weight $k$. Clearly if $f, g$ share $(a, k)$ then $f, g$ share $(a, p)$ for any integer $p$ with $0 \leq p<k$. Also we note that $f, g$ share a value $a$ IM or CM if and only if $f, g$ share $(a, 0)$ or $(a, \infty)$ respectively. 
Definition $1.2([6])$. Let $S$ be a set of distinct elements of $\mathbb{C} \cup\{\infty\}$ and $k$ be a nonnegative integer or $\infty$. Let

$$
E_{f}(S, k)=\bigcup_{a \in S} E_{k}(a ; f) .
$$

Clearly $E_{f}(S)=E_{f}(S, \infty)$ and $\bar{E}_{f}(S)=E_{f}(S, 0)$.

Improving the result of Lahiri-Banerjee [9] and Yi-Lin [17] the present author has recently proved the following result.

Theorem D ([1]). Let $S_{1}=\left\{z: z^{n}+a z^{n-1}+b=0\right\}, S_{2}=\{0\}$ and $S_{3}=\{\infty\}$, where $a, b$ are nonzero constants such that $z^{n}+a z^{n-1}+b=0$ has no multiple root and $n(\geq 4)$ is an integer. If for two nonconstant meromorphic functions $f$ and $g, E_{f}\left(S_{1}, 4\right)=E_{g}\left(S_{1}, 4\right), E_{f}\left(S_{2}, 0\right)=E_{g}\left(S_{2}, 0\right)$ and $E_{f}\left(S_{3}, \infty\right)=E_{g}\left(S_{3}, \infty\right)$ and $\Theta(\infty ; f)+\Theta(\infty ; g)>0$, then $f \equiv g$.

Now considering all the above theorems it is natural to ask the following question which is one of the motivations of the paper.

(i) What happens in Theorem $D$ if $f$ and $g$ share the set $S_{1}$ with weight 2 and 3 respectively?

Also note that to deal with the question of Gross none of the previous authors considered any further relaxation of the nature of sharing the set $S_{3}$ in Theorem C; they have all confined their investigations to the relaxation of the nature of sharing the sets $S_{1}$ and $S_{2}$ of Theorem C.

In this paper we concentrate our attention on relaxation of sharing $S_{3}$. We now state the following three theorems which are the main results of the paper.

TheOREM 1.1. Let $S_{1}=\left\{z: z^{n}+a z^{n-1}+b=0\right\}, S_{2}=\{0\}$ and $S_{3}=\{\infty\}$, where $a, b$ are nonzero constants such that $z^{n}+a z^{n-1}+b=0$ has no multiple root and $n(\geq 4)$ is an integer. If for two nonconstant meromorphic functions $f$ and $g, E_{f}\left(S_{1}, 3\right)=E_{g}\left(S_{1}, 3\right), E_{f}\left(S_{2}, 0\right)=E_{g}\left(S_{2}, 0\right)$ and $E_{f}\left(S_{3}, \infty\right)=E_{g}\left(S_{3}, \infty\right)$ and $\Theta(\infty ; f)+\Theta(\infty ; g)>\max \left\{0, \frac{20-4 n}{7 n-11}\right\}$, then $f \equiv g$.

REMARK 1.1. If $n \geq 5$ then the assertion of Theorem 1.1 is true for $\Theta(\infty ; f)+\Theta(\infty ; g)>0$.

TheOREM 1.2. Let $S_{1}, S_{2}$ and $S_{3}$ be defined as in Theorem 1.1 and $n(\geq 4)$ be an integer. If for two nonconstant meromorphic functions $f$ and $g, E_{f}\left(S_{1}, 2\right)=E_{g}\left(S_{1}, 2\right), E_{f}\left(S_{2}, 0\right)=E_{g}\left(S_{2}, 0\right)$ and $E_{f}\left(S_{3}, \infty\right)=E_{g}\left(S_{3}, \infty\right)$ and $\Theta(\infty ; f)+\Theta(\infty ; g)>\max \left\{0, \frac{32-4 n}{5 n-4}\right\}$, then $f \equiv g$.

REMARK 1.2. If $n \geq 8$ then Theorem 1.2 is true for $\Theta(\infty ; f)+\Theta(\infty ; g)>0$.

Theorem 1.3. Let $S_{1}, S_{2}$ and $S_{3}$ be defined as in Theorem 1.1 and $n(\geq 4)$ be an integer. If for two nonconstant meromorphic functions $f$ and $g$, 
$E_{f}\left(S_{1}, 4\right)=E_{g}\left(S_{1}, 4\right), E_{f}\left(S_{2}, 0\right)=E_{g}\left(S_{2}, 0\right)$ and $E_{f}\left(S_{3}, 6\right)=E_{g}\left(S_{3}, 6\right)$, and $\Theta(\infty ; f)+\Theta(\infty ; g)>0$, then $f \equiv g$.

Remark 1.3. Clearly Theorem 1.3 improves Theorem D.

The following example shows that the condition $\Theta(\infty ; f)+\Theta(\infty ; g)>0$ is not only sharp in Theorem 1.3 but also in Theorems 1.1 and 1.2 when $n \geq 5$ and $n \geq 8$ respectively.

EXAMPLE 1.1. Let

$$
g(z)=-a \frac{e^{(n-1) z}-1}{e^{n z}-1}, \quad f(z)=e^{z} g(z)
$$

and $S_{i}$ be as in Theorem 1.1. Then $E_{f}\left(S_{i}, \infty\right)=E_{g}\left(S_{i}, \infty\right)$ for $i=1,2,3$ because $f^{n-1}(f+a) \equiv g^{n-1}(g+a)$ and $f \equiv e^{z} g$. Also $\Theta(\infty ; f)+\Theta(\infty ; g)=0$ and $f \not \equiv g$.

For the standard definitions and notations of value distribution theory we refer to [4]; we now explain some specific notations used in this paper.

Definition $1.3([5])$. For $a \in \mathbb{C} \cup\{\infty\}$ we denote by $N(r, a ; f \mid=1)$ the counting function of simple $a$-points of $f$. For a positive integer $m$ we denote by $N(r, a ; f \mid \leq m)$ (resp. $N(r, a ; f \mid \geq m)$ ) the counting function of those $a$-points of $f$ whose multiplicities are not greater (resp. less) than $m$ where each $a$-point is counted according to its multiplicity.

$\bar{N}(r, a ; f \mid \leq m)$ and $\bar{N}(r, a ; f \mid \geq m)$ are defined similarly, where in counting the $a$-points of $f$ we ignore the multiplicities.

Also $N(r, a ; f \mid<m), N(r, a ; f \mid>m), \bar{N}(r, a ; f \mid<m)$ and $\bar{N}(r, a ; f \mid>m)$ are defined analogously.

Definition 1.4. We denote by $\bar{N}(r, a ; f \mid=k)$ the reduced counting function of those $a$-points of $f$ whose multiplicity is exactly $k$, where $k \geq 2$ is an integer.

DeFinition 1.5. Let $f$ and $g$ be two nonconstant meromorphic functions such that $f$ and $g$ share $(a, k)$ where $a \in \mathbb{C} \cup\{\infty\}$. Let $z_{0}$ be an $a$-point of $f$ with multiplicity $p$ and an $a$-point of $g$ with multiplicity $q$. We denote by $\bar{N}_{L}(r, a ; f)$ the counting function of those $a$-points of $f$ and $g$ where $p>q$, and by $\bar{N}_{E}^{(k+1}(r, a ; f)$ the counting function of those $a$-points of $f$ and $g$ where $p=q \geq k+1$; each point in these counting functions is counted only once. In the same way we can define $\bar{N}_{L}(r, a ; g)$ and $\bar{N}_{E}^{(k+1}(r, a ; g)$.

Definition $1.6([7])$. We set

$$
N_{2}(r, a ; f)=\bar{N}(r, a ; f)+\bar{N}(r, a ; f \mid \geq 2) .
$$

Definition $1.7([6,7])$. Let $f, g$ share a value $a$ IM. We denote by $\bar{N}_{*}(r, a ; f, g)$ the reduced counting function of those $a$-points of $f$ whose multiplicities differ from the multiplicities of the corresponding $a$-points of $g$. 
Clearly $\bar{N}_{*}(r, a ; f, g) \equiv \bar{N}_{*}(r, a ; g, f)$ and $\bar{N}_{*}(r, a ; f, g)=\bar{N}_{L}(r, a ; f)+$ $\bar{N}_{L}(r, a ; g)$.

Definition 1.8 ([10]). Let $a, b \in \mathbb{C} \cup\{\infty\}$. We denote by $N(r, a ; f \mid$ $g=b$ ) the counting function of those $a$-points of $f$, counted according to multiplicity, which are $b$-points of $g$.

Definition $1.9([10])$. Let $a, b_{1}, \ldots, b_{q} \in \mathbb{C} \cup\{\infty\}$. We denote by $N(r, a$; $\left.f \mid g \neq b_{1}, \ldots, b_{q}\right)$ the counting function of those $a$-points of $f$, counted according to multiplicity, which are not $b_{i}$-points of $g$ for $i=1, \ldots, q$.

2. Lemmas. Let $F$ and $G$ be two nonconstant meromorphic functions defined as follows:

$$
F=\frac{f^{n-1}(f+a)}{-b}, \quad G=\frac{g^{n-1}(g+a)}{-b} .
$$

We shall denote by $H, \Phi$ and $V$ the following three functions:

$$
\begin{aligned}
H & =\left(\frac{F^{\prime \prime}}{F^{\prime}}-\frac{2 F^{\prime}}{F-1}\right)-\left(\frac{G^{\prime \prime}}{G^{\prime}}-\frac{2 G^{\prime}}{G-1}\right), \\
\Phi & =\frac{F^{\prime}}{F-1}-\frac{G^{\prime}}{G-1}, \\
V & =\left(\frac{F^{\prime}}{F-1}-\frac{F^{\prime}}{F}\right)-\left(\frac{G^{\prime}}{G-1}-\frac{G^{\prime}}{G}\right)=\frac{F^{\prime}}{F(F-1)}-\frac{G^{\prime}}{G(G-1)} .
\end{aligned}
$$

Lemma 2.1 ([7, Lemma 1]). Let $F, G$ share $(1,1)$ and $H \not \equiv 0$. Then

$$
N(r, 1 ; F \mid=1)=N(r, 1 ; G \mid=1) \leq N(r, H)+S(r, F)+S(r, G) .
$$

Lemma 2.2. Let $S_{1}, S_{2}$ and $S_{3}$ be as in Theorem 1.1 and $F, G$ be given by (2.1). If for two nonconstant meromorphic functions $f$ and $g, E_{f}\left(S_{1}, 0\right)=$ $E_{g}\left(S_{1}, 0\right), E_{f}\left(S_{2}, 0\right)=E_{g}\left(S_{2}, 0\right), E_{f}\left(S_{3}, 0\right)=E_{g}\left(S_{3}, 0\right)$ and $H \not \equiv 0$, then

$$
\begin{aligned}
N(r, H) \leq & \bar{N}_{*}(r, 0, f, g)+\bar{N}(r, 0 ; f+a \mid \geq 2)+\bar{N}(r, 0 ; g+a \mid \geq 2) \\
& +\bar{N}_{*}(r, 1 ; F, G)+\bar{N}_{*}(r, \infty ; f, g)+\bar{N}_{0}\left(r, 0 ; F^{\prime}\right)+\bar{N}_{0}\left(r, 0 ; G^{\prime}\right),
\end{aligned}
$$

where $\bar{N}_{0}\left(r, 0 ; F^{\prime}\right)$ is the reduced counting function of those zeros of $F^{\prime}$ which are not the zeros of $F(F-1)$, and $\bar{N}_{0}\left(r, 0 ; G^{\prime}\right)$ is similarly defined.

Proof. Since $E_{f}\left(S_{1}, 0\right)=E_{g}\left(S_{1}, 0\right)$ it follows that $F$ and $G$ share $(1,0)$. We can easily verify that possible poles of $H$ occur at (i) those zeros of $f$ and $g$ whose multiplicities differ from the multiplicities of the corresponding zeros of $g$ and $f$ respectively, (ii) multiple zeros of $f+a$ and $g+a$, (iii) those poles of $f$ and $g$ whose multiplicities differ from the multiplicities of the corresponding poles of $g$ and $f$ respectively, (iv) 1-points of $F$ and $G$ with different multiplicities, (v) zeros of $F^{\prime}$ which are not the zeros of $F(F-1)$, (v) zeros of $G^{\prime}$ which are not the zeros of $G(G-1)$. Since $H$ has only simple poles, the lemma follows from the above. 
LEMMa 2.3 ([13]). Let $f$ be a nonconstant meromorphic function and $P(f)=a_{0}+a_{1} f+a_{2} f^{2}+\cdots+a_{n} f^{n}$, where $a_{0}, a_{1}, a_{2}, \ldots, a_{n}$ are constants and $a_{n} \neq 0$. Then $T(r, P(f))=n T(r, f)+O(1)$.

Lemma 2.4. Let $F$ and $G$ be given by (2.1). If $f, g$ share $(0,0)$ and 0 is not a Picard exceptional value of $f$ and $g$, then $\Phi \equiv 0$ implies $F \equiv G$.

Proof. Suppose $\Phi \equiv 0$. Then by integration we obtain $F-1 \equiv C(G-1)$. It is clear that if $z_{0}$ is a zero of $f$ then it is a zero of $g$. So from (2.1) it follows that $F\left(z_{0}\right)=0$ and $G\left(z_{0}\right)=0$. So $C=1$ and hence $F \equiv G$.

Lemma 2.5. Let $F$ and $G$ be given by (2.1), $n \geq 3$ an integer and $\Phi \not \equiv 0$. If $F, G$ share $(1, m)$ and $f, g$ share $(0, p)$ and $(\infty, k)$, where $0 \leq p<\infty$, then

$$
\begin{aligned}
{[(n-1) p+n-2] \bar{N}(r, 0 ; f \mid \geq p+1) \leq } & \bar{N}_{*}(r, 1 ; F, G)+\bar{N}_{*}(r, \infty ; F, G) \\
& +S(r, f)+S(r, g) .
\end{aligned}
$$

Proof. If 0 is an e.v.P. (Picard exceptional value) of $f$ and $g$ then the assertion follows immediately.

Next suppose 0 is not an e.v.P. of $f$ and $g$. Let $z_{0}$ be a zero of $f$ with multiplicity $q$ and a zero of $g$ with multiplicity $r$. From (2.1) we know that $z_{0}$ is a zero of $F$ with multiplicity $(n-1) q$ and a zero of $G$ with multiplicity $(n-1) r$. We note that $F$ and $G$ have no zero of multiplicity $t$ where $(n-1) p<$ $t<(n-1)(p+1)$. So from the definition of $\Phi$ it is clear that $z_{0}$ is a zero of $\Phi$ with multiplicity at least $(n-1)(p+1)-1$. So we have

$$
\begin{aligned}
{[(n-1) p+n-2] \bar{N}(r, 0 ; f \mid \geq p+1) } \\
=[(n-1) p+n-2] \bar{N}(r, 0 ; g \mid \geq p+1) \\
=[(n-1) p+n-2] \bar{N}(r, 0 ; F \mid \geq(n-1)(p+1)) \\
\leq N(r, 0 ; \Phi) \leq N(r, \infty ; \Phi)+S(r, f)+S(r, g) \\
\leq \bar{N}_{*}(r, \infty ; F, G)+\bar{N}_{*}(r, 1 ; F, G)+S(r, f)+S(r, g) .
\end{aligned}
$$

Lemma 2.6. Let $F$ and $G$ be given by (2.1), and suppose $f, g$ share $(\infty, 0)$ and $\infty$ is not an Picard exceptional value of $f$ and $g$. Then $V \equiv 0$ implies $F \equiv G$.

Proof. Suppose $V \equiv 0$. Then by integration we obtain

$$
1-\frac{1}{F} \equiv A\left(1-\frac{1}{G}\right) \text {. }
$$

It is clear that if $z_{0}$ is a pole of $f$ then it is a pole of $g$. Hence from the definition of $F$ and $G$ we have $1 / F\left(z_{0}\right)=0$ and $1 / G\left(z_{0}\right)=0$. So $A=1$ and hence $F \equiv G$. 
Lemma 2.7. Let $F, G$ be given by (2.1) and $V \not \equiv 0$. If $f$, $g$ share $(0,0)$ and $(\infty, k)$, where $0 \leq k<\infty$, and $F, G$ share $(1, m)$, then the poles of $F$ and $G$ are the zeros of $V$ and

$$
\begin{aligned}
(n k+n-1) \bar{N}(r, \infty ; f \mid \geq k+1)= & (n k+n-1) \bar{N}(r, \infty ; g \mid \geq k+1) \\
\leq & \bar{N}_{*}(r, 0 ; f, g)+\bar{N}(r, 0 ; f+a) \\
& +\bar{N}(r, 0 ; g+a)+\bar{N}_{L}(r, 1 ; F) \\
& +\bar{N}_{L}(r, 1 ; G)+S(r, f)+S(r, g) .
\end{aligned}
$$

Proof. If $\infty$ is an e.v.P. of $f$ and $g$ then the assertion follows immediately.

Next suppose $\infty$ is not an e.v.P. of $f$ and $g$. Since $f, g$ share $(\infty, k)$, it follows that $F, G$ share $(\infty, n k)$ and so a pole of $F$ with multiplicity $p(\geq n k+1)$ is a pole of $G$ with multiplicity $r(\geq n k+1)$ and vice versa. We note that $F$ and $G$ have no pole of multiplicity $q$ where $n k<q<n k+n$. So using Lemma 2.3 and noting that $f, g$ share $(0,0)$ and $F, G$ share $(1, m)$ we get, from the definition of $V$,

$$
\begin{aligned}
(n k+n-1) \bar{N}(r, \infty ; & f \mid \geq k+1) \\
= & (n k+n-1) \bar{N}(r, \infty ; g \mid \geq k+1) \\
= & (n k+n-1) \bar{N}(r, \infty ; F \mid \geq n k+n) \\
\leq & N(r, 0 ; V) \leq N(r, \infty ; V)+S(r, f)+S(r, g) \\
\leq & \bar{N}_{*}(r, 0 ; f, g)+\bar{N}(r, 0 ; f+a)+\bar{N}(r, 0 ; g+a) \\
& +\bar{N}_{*}(r, 1 ; F, G)+S(r, f)+S(r, g) .
\end{aligned}
$$

LEMma 2.8 ([1, Lemma 3$])$. Let $f$ and $g$ be two nonconstant meromorphic functions sharing $(1, m)$, where $2 \leq m<\infty$. Then

$$
\begin{aligned}
\bar{N}(r, 1 ; f \mid=2)+2 \bar{N}(r, 1 ; f \mid=3)+\cdots+(m-1) \bar{N}(r, 1 ; f \mid=m) & \\
+m \bar{N}_{L}(r, 1 ; f)+(m+1) \bar{N}_{L}(r, 1 ; g) & +m \bar{N}_{E}^{(m+1}(r, 1 ; f) \\
& \leq N(r, 1 ; g)-\bar{N}(r, 1 ; g) .
\end{aligned}
$$

Lemma 2.9. Let $F, G$ be given by (2.1) and suppose they share $(1, m)$, where $2 \leq m<\infty$. If $f, g$ share $(0, p)$ and $(\infty, k)$, and $H \not \equiv 0$, then

$$
\begin{aligned}
T(r, F) \leq & \bar{N}(r, 0 ; f)+\bar{N}(r, 0 ; g)+\bar{N}_{*}(r, 0 ; f, g)+N_{2}(r, 0 ; f+a) \\
& +N_{2}(r, 0 ; g+a)+\bar{N}(r, \infty ; f)+\bar{N}(r, \infty ; g) \\
& +\bar{N}_{*}(r, \infty ; f, g)-m(r, 1 ; G)-\bar{N}(r, 1 ; F \mid=3) \\
& -\cdots-(m-2) \bar{N}(r, 1 ; F \mid=m)-(m-2) \bar{N}_{L}(r, 1 ; F) \\
& -(m-1) \bar{N}_{L}(r, 1 ; G)-(m-1) \bar{N}_{E}^{(m+1}(r, 1 ; F) \\
& +S(r, F)+S(r, G) .
\end{aligned}
$$


Proof. By the second fundamental theorem we get

$$
\begin{aligned}
T(r, F)+T(r, G) \leq & \bar{N}(r, 0 ; F)+\bar{N}(r, \infty ; F)+\bar{N}(r, 0 ; G) \\
& +\bar{N}(r, \infty ; G)+\bar{N}(r, 1 ; F)+\bar{N}(r, 1 ; G) \\
& -N_{0}\left(r, 0 ; F^{\prime}\right)-N_{0}\left(r, 0 ; G^{\prime}\right)+S(r, F)+S(r, G) .
\end{aligned}
$$

In view of Definition 1.7, using Lemmas 2.1, 2.2 and 2.8 we see that

$$
\begin{aligned}
\bar{N}(r, 1 ; & F)+\bar{N}(r, 1 ; G) \\
\leq & N(r, 1 ; F \mid=1)+\bar{N}(r, 1 ; F \mid=2)+\bar{N}(r, 1 ; F \mid=3) \\
& +\cdots+\bar{N}(r, 1 ; F \mid=m)+\bar{N}_{E}^{(m+1}(r, 1 ; F) \\
& +\bar{N}_{L}(r, 1 ; F)+\bar{N}_{L}(r, 1 ; G)+\bar{N}(r, 1 ; G) \\
\leq & \bar{N}_{*}(r, 0 ; f, g)+\bar{N}(r, 0 ; f+a \mid \geq 2) \\
& +\bar{N}(r, 0 ; g+a \mid \geq 2)+\bar{N}_{*}(r, \infty ; f, g)+\bar{N}_{L}(r, 1 ; F) \\
& +\bar{N}_{L}(r, 1 ; G)+\bar{N}(r, 1 ; F \mid=2)+\cdots \\
& \left.+\bar{N}_{(r, 1 ; F} \mid=m\right)+\bar{N}_{E}^{(m+1}(r, 1 ; F) \\
& +\bar{N}_{L}(r, 1 ; F)+\bar{N}_{L}(r, 1 ; G)+T(r, G)-m(r, 1 ; G) \\
& +O(1)-\bar{N}(r, 1 ; F \mid=2)-2 \bar{N}(r, 1 ; F \mid=3) \\
& -(m-1) \bar{N}(r, 1 ; F \mid=m)-\cdots-m \bar{N}_{E}^{(m+1}(r, 1 ; F) \\
& -m \bar{N}_{L}(r, 1 ; F)-(m+1) \bar{N}_{L}(r, 1 ; G)+\bar{N}_{0}\left(r, 0 ; F^{\prime}\right) \\
& +\bar{N}_{0}\left(r, 0 ; G^{\prime}\right)+S(r, F)+S(r, G) \\
\leq & \bar{N}_{*}(r, 0 ; f, g)+\bar{N}(r, 0 ; f+a \mid \geq 2)+\bar{N}(r, 0 ; g+a \mid \geq 2) \\
& +\bar{N}_{*}(r, \infty ; f, g)+T(r, G)-m(r, 1 ; G)-\bar{N}_{(r, 1 ; F \mid=3)} \\
& \left.-2 \bar{N}_{(r, 1 ; F} \mid=4\right)-\cdots-(m-2) \bar{N}(r, 1 ; F \mid=m) \\
& -(m-2) \bar{N}_{L}(r, 1 ; F)-(m-1) \bar{N}_{L}(r, 1 ; G) \\
& -(m-1) \bar{N}\left(m+1(r, 1 ; F)+\bar{N}_{0}\left(r, 0 ; F^{\prime}\right)+\bar{N}_{0}\left(r, 0 ; G^{\prime}\right)\right. \\
& +S(r, F)+S(r, G) .
\end{aligned}
$$

From (2.2) and (2.3) in view of Definition 1.6 the lemma follows.

Lemma 2.10 ([9, Lemma 3]). Let $f, g$ be two nonconstant meromorphic functions sharing $(0, \infty)$ and $(\infty, \infty)$, and $\Theta(\infty ; f)+\Theta(\infty ; g)>0$. Then $f^{n-1}(f+a) \equiv g^{n-1}(g+a)$ implies $f \equiv g$, where $n(\geq 2)$ is an integer and $a$ is a nonzero finite constant. 
LEMma 2.11 ([8, Lemma 5]). If two nonconstant meromorphic functions $f, g$ share $(\infty, 0)$, then for $n \geq 2$,

$$
f^{n-1}(f+a) g^{n-1}(g+a) \not \equiv b^{2}
$$

where $a, b$ are finite nonzero constants.

Lemma 2.12 ([16, Lemma 6$])$. If $H \equiv 0$, then $F, G$ share $(1, \infty)$. If further $F, G$ share $(\infty, 0)$, then they share $(\infty, \infty)$.

Lemma $2.13([11])$. If $N\left(r, 0 ; f^{(k)} \mid f \neq 0\right)$ denotes the counting function of those zeros of $f^{(k)}$ which are not the zeros of $f$, where a zero of $f^{(k)}$ is counted according to its multiplicity, then

$$
\begin{aligned}
N\left(r, 0 ; f^{(k)} \mid f \neq 0\right) \leq & k \bar{N}(r, \infty ; f)+N(r, 0 ; f \mid<k) \\
& +k \bar{N}(r, 0 ; f \mid \geq k)+S(r, f) .
\end{aligned}
$$

Lemma 2.14. Let $F, G$ be given by (2.1) and suppose $F, G$ share $(1, m)$, $0 \leq m<\infty$ and $\omega_{1}, \ldots, \omega_{n}$ are the distinct roots of the equation $z^{n}+a z^{n-1}$ $+b=0$ and $n \geq 3$. Then

$$
\bar{N}_{L}(r, 1 ; F) \leq \frac{1}{m+1}\left[\bar{N}(r, 0 ; f)+\bar{N}(r, \infty ; f)-N_{\otimes}\left(r, 0 ; f^{\prime}\right)\right]+S(r, f),
$$

where $N_{\otimes}\left(r, 0 ; f^{\prime}\right)=N\left(r, 0 ; f^{\prime} \mid f \neq 0, \omega_{1}, \ldots, \omega_{n}\right)$.

Proof. Using Lemmas 2.3 and 2.13 we see that

$$
\begin{aligned}
\bar{N}_{L}(r, 1 ; F) & \leq \bar{N}(r, 1 ; F \mid \geq m+2) \\
& \leq \frac{1}{m+1}(N(r, 1 ; F)-\bar{N}(r, 1 ; F)) \\
& \leq \frac{1}{m+1} \sum_{j=1}^{n}\left(N\left(r, \omega_{j} ; f\right)-\bar{N}\left(r, \omega_{j} ; f\right)\right) \\
& \leq \frac{1}{m+1}\left(N\left(r, 0 ; f^{\prime} \mid f \neq 0\right)-N_{\otimes}\left(r, 0 ; f^{\prime}\right)\right) \\
& \leq \frac{1}{m+1}\left[\bar{N}(r, 0 ; f)+\bar{N}(r, \infty ; f)-N_{\otimes}\left(r, 0 ; f^{\prime}\right)\right]+S(r, f) .
\end{aligned}
$$

Lemma 2.15. Under the assumptions of Lemma 2.14,

$$
\bar{N}_{*}(r, 1 ; F, G) \leq \frac{1}{m}\left[\bar{N}(r, 0 ; f)+\bar{N}(r, \infty ; f)-N_{\otimes}\left(r, 0 ; f^{\prime}\right)\right]+S(r, f) .
$$

Proof. Since

$$
\bar{N}_{*}(r, 1 ; F, G) \leq \bar{N}(r, 1 ; F \mid \geq m+1) \leq \frac{1}{m}(N(r, 1 ; F)-\bar{N}(r, 1 ; F)),
$$

the proof can be carried out along the lines of the proof of Lemma 2.14. 
Lemma 2.16 ([14]). Let $F, G$ be two nonconstant meromorphic functions sharing $(1, \infty)$ and $(\infty, \infty)$. If

$$
N_{2}(r, 0 ; F)+N_{2}(r, 0 ; F)+2 \bar{N}(r, \infty ; F)<\lambda T_{1}(r)+S_{1}(r),
$$

where $\lambda<1$ and $T_{1}(r)=\max \{T(r, F), T(r, G)\}$ and $S_{1}(r)=o\left(T_{1}(r)\right)$, $r \rightarrow \infty$, outside a possible exceptional set of finite linear measure, then $F \equiv G$ or $F G \equiv 1$.

Lemma 2.17. Let $F, G$ be given by (2.1) and $n \geq 4$, and suppose that $F, G$ share $(1, m)$, where $2 \leq m<\infty$. If $f, g$ share $(0,0)$ and $(\infty, k)$, and $\Theta(\infty ; f)+\Theta(\infty ; g)>0$ and $H \equiv 0$, then $f \equiv g$.

Proof. Since $H \equiv 0$, Lemma 2.12 shows that $F, G$ share $(1, \infty)$ and $(\infty, \infty)$. If possible, suppose $F \not \equiv G$. Then from Lemmas 2.4 and 2.5 we have

$$
\bar{N}(r, 0 ; f)=\bar{N}(r, 0 ; g)=S(r) .
$$

Again from Lemmas 2.6 and 2.7 we obtain

$$
\bar{N}(r, \infty ; f)+\bar{N}(r, \infty ; g) \leq \frac{4}{n-1} T(r)+S(r) .
$$

Therefore

$$
\begin{aligned}
N_{2}(r, 0 ; F)+N_{2}(r, 0 ; G) & +2 \bar{N}(r, \infty ; F) \\
\leq & 2 \bar{N}(r, 0 ; f)+2 \bar{N}(r, 0 ; g)+N_{2}(r, 0 ; f+a) \\
& +N_{2}(r, 0 ; g+a)+2 \bar{N}(r, \infty ; f) \\
\leq & N_{2}(r, 0 ; f+a)+N_{2}(r, 0 ; g+a) \\
& +\bar{N}(r, \infty ; f)+\bar{N}(r, \infty ; g)+S(r) .
\end{aligned}
$$

Using Lemma 2.3 we obtain

$$
T_{1}(r)=n \max \{T(r, f), T(r, g)\}+O(1)=n T(r)+O(1) .
$$

So again using Lemma 2.3 we get from (2.4) and (2.5),

$$
N_{2}(r, 0 ; F)+N_{2}(r, 0 ; G)+2 \bar{N}(r, \infty ; F) \leq \frac{2+\frac{4}{n-1}}{n} T_{1}(r)+S_{1}(r) .
$$

Since $\frac{2+\frac{4}{n-1}}{n}<1$ for $n \geq 4$, Lemma 2.16 yields $F G \equiv 1$, which is impossible by Lemma 2.11 . Hence $F \equiv G$, i.e. $f^{n-1}(f+a) \equiv g^{n-1}(g+a)$. This together with the assumption that $f$ and $g$ share $(0,0)$ implies that $f$ and $g$ share $(0, \infty)$. Now the lemma follows from Lemma 2.10 .

\section{Proofs of the theorems}

Proof of Theorem 1.1. Let $F, G$ be given by (2.1). Then $F$ and $G$ share $(1,3)$ and $(\infty, \infty)$. We consider the following cases. 
Case 1: $H \not \equiv 0$. Then $F \not \equiv G$. Suppose 0 is not an e.v.P. of $f$ and $g$. Then by Lemma 2.4 we get $\Phi \not \equiv 0$. Noting that $f$ and $g$ sharing $(0,0)$ implies $\bar{N}_{*}(r, 0 ; f, g) \leq \bar{N}(r, 0 ; f)=\bar{N}(r, 0 ; g)$, from Lemmas $2.3,2.5,2.9$ and 2.14 we obtain, for $\varepsilon>0$,

$$
\begin{aligned}
n T(r, f) \leq & 3 \bar{N}(r, 0 ; f)+N_{2}(r, 0 ; f+a)+N_{2}(r, 0 ; g+a) \\
& +\bar{N}(r, \infty ; f)+\bar{N}(r, \infty ; g)-\bar{N}_{L}(r, 1 ; F) \\
& -2 \bar{N}_{L}(r, 1 ; G)+S(r, f)+S(r, g) \\
\leq & 3 \bar{N}(r, 0 ; f)+T(r, f)+T(r, g)+\bar{N}(r, \infty ; f) \\
& +\bar{N}(r, \infty ; g)-\bar{N}_{L}(r, 1 ; F)-2 \bar{N}_{L}(r, 1 ; G) \\
& +S(r, f)+S(r, g) \\
\leq & \frac{3}{n-2}\left[\bar{N}_{L}(r, 1 ; F)+\bar{N}_{L}(r, 1 ; G)\right]+2 T(r) \\
& +\bar{N}(r, \infty ; f)+\bar{N}(r, \infty ; g)-\bar{N}_{L}(r, 1 ; F) \\
& -2 \bar{N}_{L}(r, 1 ; G)+S(r, f)+S(r, g) \\
\leq & \frac{5-n}{4(n-2)}[\bar{N}(r, 0 ; f)+\bar{N}(r, \infty ; f)]+2 T(r) \\
& +\bar{N}(r, \infty ; f)+\bar{N}(r, \infty ; g)+S(r, f)+S(r, g) \\
\leq & \frac{5-n}{4(n-2)} T(r, f)+2 T(r)+\left(2+\frac{5-n}{4(n-2)}\right) \bar{N}(r, \infty ; f) \\
& +S(r) \\
\leq & {\left[4+\frac{5-n}{2(n-2)}-\frac{7 n-11}{8(n-2)}\{\Theta(\infty ; f)+\Theta(\infty ; g)-2 \varepsilon\}\right] T(r) } \\
& +S(r) .
\end{aligned}
$$

If 0 is an e.v.P. of $f$ and $g$ then (3.1) holds automatically.

In the same way we can obtain

$$
\begin{aligned}
n T(r, g) \leq & {\left[4+\frac{5-n}{2(n-2)}-\frac{7 n-11}{8(n-2)}\{\Theta(\infty ; f)+\Theta(\infty ; g)-2 \varepsilon\}\right] T(r) } \\
& +S(r) .
\end{aligned}
$$

Combining (3.1) and (3.2) we see that

$$
\left[n-4-\frac{5-n}{2(n-2)}+\frac{7 n-11}{8(n-2)}\{\Theta(\infty ; f)+\Theta(\infty ; g)-2 \varepsilon\}\right] T(r) \leq S(r) .
$$

Since $\Theta(\infty ; f)+\Theta(\infty ; g)>\max \left\{0, \frac{20-4 n}{7 n-11}\right\}$, there exists a $\delta>0$ such that

$$
\Theta(\infty ; f)+\Theta(\infty ; g)=\max \left\{0, \frac{20-4 n}{7 n-11}\right\}+\delta .
$$

If we choose $0<\varepsilon<\delta / 2$ then (3.2) leads to a contradiction. 
CASE 2: $H \equiv 0$. Then the assertion follows from Lemma 2.17.

Proof of Theorem 1.2. Let $F, G$ be given by (2.1). Then $F$ and $G$ share $(1,2)$ and $(\infty, \infty)$. We consider the following cases.

CASe 1: $H \not \equiv 0$. Then $F \not \equiv G$. Suppose 0 is not an e.v.P. of $f$ and $g$. Then by Lemma 2.4 we get $\Phi \not \equiv 0$. So from Lemmas 2.3, 2.5, 2.9 and 2.14 we obtain, for $\varepsilon>0$,

$$
\begin{aligned}
n T(r, f) \leq & 3 \bar{N}(r, 0 ; f)+N_{2}(r, 0 ; f+a)+N_{2}(r, 0 ; g+a) \\
& +\bar{N}(r, \infty ; f)+\bar{N}(r, \infty ; g)-\bar{N}_{L}(r, 1 ; G) \\
& +S(r, f)+S(r, g) \\
\leq & \frac{3}{n-2} \bar{N}_{L}(r, 1 ; F)+\frac{5-n}{n-2} \bar{N}_{L}(r, 1 ; G)+2 T(r) \\
& +\bar{N}(r, \infty ; f)+\bar{N}(r, \infty ; g)+S(r, f)+S(r, g) \\
\leq & \left(2+\frac{8-n}{3(n-2)}\right) T(r)+\left(2+\frac{8-n}{3(n-2)}\right) \bar{N}(r, \infty ; f)+S(r) \\
\leq & {\left[4+\frac{16-2 n}{3(n-2)}-\frac{5 n-4}{6(n-2)}\{\Theta(\infty ; f)+\Theta(\infty ; g)-2 \varepsilon\}\right] T(r) } \\
& +S(r) .
\end{aligned}
$$

If 0 is an e.v.P. of $f$ and $g$ then (3.3) holds automatically.

In the same manner we can obtain

$$
\begin{aligned}
n T(r, g) \leq & {\left[4+\frac{16-2 n}{3(n-2)}-\frac{5 n-4}{6(n-2)}\{\Theta(\infty ; f)+\Theta(\infty ; g)-2 \varepsilon\}\right] T(r) } \\
& +S(r) .
\end{aligned}
$$

Combining (3.3) and (3.4) we see that

$$
\left[n-4-\frac{16-2 n}{3(n-2)}+\frac{5 n-4}{6(n-2)}\{\Theta(\infty ; f)+\Theta(\infty ; g)-2 \varepsilon\}\right] T(r) \leq S(r),
$$

which leads to a contradiction for arbitrary $\varepsilon>0$.

CASE $2: H \equiv 0$. Then the assertion follows from Lemma 2.17.

Proof of Theorem 1.3. Let $F, G$ be given by (2.1). Then $F$ and $G$ share $(1,4)$ and $(\infty, 6 n)$. We consider the following cases.

Case 1: $H \not \equiv 0$. Then $F \not \equiv G$. Suppose $0, \infty$ are not Picard exceptional values of $f$ and $g$. Then by Lemmas 2.4 and 2.6 we get $\Phi \not \equiv 0$ and $V \not \equiv 0$. Noting that $f, g$ sharing $(0,0)$ and $(\infty, 6)$ implies $\bar{N}_{*}(r, 0 ; f, g) \leq$ $\bar{N}(r, 0 ; f)=\bar{N}(r, 0 ; g)$ and $\bar{N}_{*}(r, \infty ; f, g) \leq \bar{N}(r, \infty ; f \mid \geq 7)=\bar{N}(r, \infty ; g \mid \geq 7)$, from Lemmas 2.3, 2.5 and 2.9 we obtain 


$$
\begin{aligned}
n T(r, f) & +n T(r, g) \\
\leq & 6 \bar{N}(r, 0 ; f)+2 T(r, f)+2 T(r, g)+4 \bar{N}(r, \infty ; f) \\
& +2 \bar{N}(r, \infty ; f \mid \geq 7)-5 \bar{N}_{*}(r, 1 ; F, G)+S(r, f)+S(r, g) \\
\leq & 2 T(r, f)+2 T(r, g)+\left\{2+\frac{6}{n-2}\right\} \bar{N}(r, \infty ; f \mid \geq 7) \\
& +\frac{6}{n-2} \bar{N}_{*}(r, 1 ; F, G)+4 \bar{N}(r, \infty ; f) \\
& -5 \bar{N}_{*}(r, 1 ; F, G)+S(r, f)+S(r, g) .
\end{aligned}
$$

So using respectively Lemma 2.7 for $k=6$ and $k=0$, Lemma 2.5 for $p=0$ and Lemma 2.15 we deduce from (3.5) that

$$
\begin{aligned}
& n T(r, f)+n T(r, g) \\
\leq & \left(2+\frac{3(n+1)}{(n-2)(7 n-1)}\right)\{T(r, f)+T(r, g)\} \\
& +\left(\frac{6}{n-2}+\frac{2(n+1)}{(n-2)(7 n-1)}\right) \bar{N}_{*}(r, 1 ; F, G) \\
& +\frac{4}{n-1}\left[T(r, f)+T(r, g)+\bar{N}(r, 0 ; f)+\bar{N}_{*}(r, 1 ; F, G)\right] \\
& -5 \bar{N}_{*}(r, 1 ; F, G)+S(r, f)+S(r, g) \\
\leq & \left(2+\frac{4}{n-1}+\frac{3(n+1)}{(n-2)(7 n-1)}\right)\{T(r, f)+T(r, g)\} \\
& +\left(\frac{6}{n-2}+\frac{4}{n-1}+\frac{2(n+1)}{(n-2)(7 n-1)}\right) \bar{N}_{*}(r, 1 ; F, G) \\
& +\frac{4}{(n-1)(n-2)}\left[\bar{N}(r, \infty ; f \mid \geq 7)+\bar{N}_{*}(r, 1 ; F, G)\right]-5 \bar{N}_{*}(r, 1 ; F, G) \\
& +S(r, f)+S(r, g) \\
\leq & \left(2+\frac{4 n-6}{(n-1)(n-2)}+\frac{3(n+1)}{(n-2)(7 n-1)}\right)\{T(r, f)+T(r, g)\} \\
& +\left(\frac{10}{n-2}+\frac{2(n+1)}{(n-2)(7 n-1)}-5\right) \bar{N}_{*}(r, 1 ; F, G) \\
& +S(r, f)+S(r, g) \\
\leq & \left(2+\frac{(4 n-6)}{(n-1)(n-2)}+\frac{7(n+1)}{2(n-2)(7 n-1)}\right)\{T(r, f)+T(r, g)\} \\
& +S(r, f)+S(r, g) .
\end{aligned}
$$

From (3.6) we get a contradiction for $n \geq 4$.

If $0, \infty$ are e.v.P. of $f$ and $g$ then (3.6) holds automatically. 
CASE $2: H \equiv 0$. Now the assertion follows from Lemma 2.17.

\section{References}

[1] A. Banerjee, On a question of Gross, J. Math. Anal. Appl. 327 (2007) 1273-1283.

[2] M. Fang and W. Xu, A note on a problem of Gross, Chinese J. Contemp. Math. 18 (1997), 395-402.

[3] F. Gross, Factorization of meromorphic functions and some open problems, in: Complex Analysis (Lexington, KY, 1976), Lecture Notes in Math. 599, Springer, Berlin, $51-67$.

[4] W. K. Hayman, Meromorphic Functions, Clarendon Press, Oxford, 1964.

[5] I. Lahiri, Value distribution of certain differential polynomials, Int. J. Math. Math. Sci. 28 (2001), 83-91.

[6] -, Weighted sharing and uniqueness of meromorphic functions, Nagoya Math. J. 161 (2001), 193-206.

[7] - Weighted value sharing and uniqueness of meromorphic functions, Complex Var. Theory Appl. 46 (2001), 241-253.

[8] -, On a question of Hong Xun Yi, Arch. Math. (Brno) 38 (2002), 119-128.

[9] I. Lahiri and A. Banerjee, Uniqueness of meromorphic functions with deficient poles, Kyungpook Math. J. 44 (2004), 575-584.

[10] -, -, Weighted sharing of two sets, ibid. 46 (2006), 79-87.

[11] I. Lahiri and S. Dewan, Value distribution of the product of a meromorphic function and its derivative, Kodai Math. J. 26 (2003), 95-100.

[12] H. Qiu and M. Fang, A unicity theorem for meromorphic functions, Bull. Malaysian Math. Sci. Soc. 25 (2002), 31-38.

[13] C. C. Yang, On deficiencies of differential polynomials II, Math. Z. 125 (1972), $107-112$.

[14] H. X. Yi, Meromorphic functions that share one or two values, Complex Var. Theory Appl. 28 (1995), 1-11.

[15] - Meromorphic functions that share three sets, Kodai Math. J. 20 (1997), 22-32.

[16] - Meromorphic functions that share one or two values II, ibid. 22 (1999), 264-272.

[17] H. X. Yi and W. C. Lin, Uniqueness theorems concerning a question of Gross, Proc. Japan Acad. Ser. A 80 (2004), 136-140.

[18] H. X. Yi and L. Z. Yang, Meromorphic functions that share two sets, Kodai Math. J. 20 (1997), 127-134.

Department of Mathematics

Kalyani Government Engineering College

West Bengal 741235, India

E-mail: abanerjee_kal@yahoo.co.in abanerjee_kal@rediffmail.com abanerjee@mail15.com

Received 23.5.2007

and in final form 3.8.2007 